\title{
Desktop Agent Manager (DAM): Decision Mechanism
}

\author{
Deborah Ann Guerrera Ercolini \\ The MITRE Corporation, Bedford, Massachusetts
}

\author{
Mieczyslaw M. Kokar \\ Department of Electrical and Computer Engineering, Northeastern University
}

\begin{abstract}
Desktop software agents are background processes that notify a computer user of certain predefined events. The complexity of desktop computing is increasing due to the proliferation of software agents. A desktop agent manager (DAM) may become an important component of desktop computing. This article focuses on the decision mechanism for the DAM to decide which agents should be allowed to access the user, which agents' results should be stored for future review by the user, and which of the agents should be filtered out. We prove the validity of the concept of a DAM by developing an architecture that includes both a prototype of the DAM and a simulator of various types of agents. In this article, we present the results of our simulations and analyses of the DAM decisior mechanism.
\end{abstract}

\section{INTRODUCTION}

There are many applications that reside on the typical office computer: E-mail, word processor, spreadsheet, personal database manager, corporate database manager, scheduler, and so on. Many components of the shelf (COTS) have software agents as components. Typically, a software agent is defined as an autonomous background process that communicates with both the user and other programs. Sometimes agents can be configured by end users (the application provides a simple user interface for the user to describe their needs), and at other times agents need to be developed by system programmers.

Deborah Ann Guerrera Ercolini is employed by The MTTRE Corporation. The views expressed in this article are the author's and are not intended to represent in any way The MIRE Cornoration or its opinions on this subject matter.

Deborah Ann Guerrera Ercolini wishes to thank former colleague Beth Lavender for her invaluable contributions to the research described in this atticle.

Requests for reprints should be sent to Mieczyslaw M. Kokar, Department of Electrical and Computer Engineering, Northeastem University, 360 Huntington Avenue, Boston, MA 02115. E-mail; кокаrøicoe.neu.edu. 
With the increased utilization of software agents comes distraction. Agents interrupt workflow as they query for user feedback. The interruptions can be random, scheduled, ad hoc, or triggered. These events and interruptions can happen while the user is writing a document, watching a class or lecture (via video on desktop), or participating in an electronic meeting (via screen sharing, video teleconferencing). This article addresses the solution to the problem of many agents interrupting the user's workflow.

Consider the following events that could be produced by various agents and appear on the desktop at any one time:

- Notifier (your automatic query is finished; your 10:00 a.m. meeting has been changed to 11:00 a.m.; E-mail is in your in basket).

- Workflow notifier (sign this purchase requisition within $24 \mathrm{hr}$; review this document by February 22, 1994).

- Yellow or red flag notifier (your budget is overrunning; schedule is slipping).

- Alarm (printer is out of paper, final report is a month late).

- Urgent request via desktop video teleconferencing (prepare a one-page write-up for annual report on collaborative computing for specialty group).

- Network load monitor (load is low).

- Pseudo-monitor (you may have CNN running in the background to watch the late-breaking news stories, weather, etc.).

There are many examples of COTS packages that incorporate agents as components of their applications. For example, BeyondMail (Miley, 1993), an E-mail system, can filter messages by the sender of the message, by the subject, or by certain keywords in the body of the message. The user can specify to filter the messages into a particular folder or to automatically forward a message to someone else. Open Sesame! (Streeter, 1993), from Charles River Analytics Incorporated, provides intelligent agents that mimic the user's repetitive general desktop movements, such as opening and closing applications and files. This product uses neural networks and expert-system knowledge bases to achieve this performance. Magnet from No Hands Software (Tessler, 1993) incorporates intelligent agents for searching files on Macintosh network systems. These agents can be scheduled to run at specific times or can be triggered by specific events.

There are at least two possible strategies that can be used in the solution to the problem of coordination of multiple agents communicating with the user (Bird, 1993): communication-based (distributed) or centralized. In the distributed approach, particular agents would have to come to an agreement on who should be allowed to access the user and who should not through a rather extensive communication process. There are at least two problems with this kind of approach. First, agents developed by many vendors do not have a common standard communication protocol, and therefore to implement this approach, either a new standard would have to be imposed, or interfaces would have to be developed for all the kinds of agents. Second, for the user to be able to interact with all the agents in a similar manner, all the agents would have to have a common mechanism that the user could use to establish a user access control policy. For these two reasons, the 
distributed solution is very difficult to implement. More discussion of distributed heterogeneous agents can be found in Adler, Durfee, Huhns, Punch, and Simoudis (1992). In the centralized approach, one agent would have to be designated as a coordinator of all other agents. The user would need to inferact only with this agent to establish or change the user access policy. This seems to be a more feasible approach to the coordination of multiple agents. For this reason, in our research we focused on such a central agent coordinator, which we call the desktop agent manager (DAM). Our focus is on heterogeneous agents working on unrelated tasks and therefore communication among agents is of a lesser importance. The need for a user interface agent was also identified by Avouris, van Liedekerke, Lekkas, and Hall (1993) for a collection of homogeneous agents working on a common task.

The goal of our research (Ercolini, 1994) was to investigate the concept of a DAM. Toward this goal, we first performed a literature search into the existing solutions. A brief overview of existing solutions is presented in Section 2. Our first objective was to understand the requirements for such an agent manager. Our second objective was to find out whether either a solution that implements our requirements already exists, or identify such solutions that could be modified to fulfill the DAM requirements. Because none of the existing solutions satisfied our requirements, we decided to develop a DAM prototype. For this we had to define all the necessary functional components of a DAM, the architecture of the DAM, and the communication protocol to communicate with the user and with the managed agents. Additionally, we had to develop an environment to simulate various agents. From the user's point of view, the main functional element of the DAM is the filtering mechanism that makes a decision on whether an agent should report to the user or not. This article addresses the decision mechanism proposed by Ercolini (1994); it is described in Section 3. The whole architecture is described in Section 4. To evaluate the decision mechanism, we developed the simulation described in Section 5 . The results of simulations with the DAM are described in Section 6. In Section 7, we present the analysis of the simulation results. Section 8 contains conclusions and suggestions for further research in this area.

\section{EXISTING SOLUTIONS}

There are many ongoing research efforts focused on software agents. Some efforts are focused on the architecture of the agents, some are focused on how agents interact, and others focus on how agents are made to be intelligent. No solutions were found that addressed how to manage software agents for the user in the manner proposed. However, several related research efforts could potentially provide solutions if additional functionality or investigation was done.

The Envoy Framework (Palaniappan et al., 1992) includes two management components: a Bureau Chief, who manages envoys (keeps track of user's envoys, creates new envoys, assigns new tasks to envoys, and keeps track of envoy-aware applications) and a Mission Summary, the user interface to Bureau Chief and envoys, which displays the status of the envoys and their reports, and allows the user to cancel or stop an envoy. Either of these components could potentially be 
modified to provide a solution to managing the agents. Our research can be viewed as an extension to the Envoy Framework research. Our DAM is very similar to Mission Summary, in that it keeps track of the existing agents and what tasks they are performing. Envoy research indicated that users wanted a more sophisticated Mission Summary. Our solution takes Mission Summary manager one step further. The DAM has a decision mechanism to decide whether an agent can display its results to the user.

The Object Management Group (OMG) is providing an architecture for distributed, cross-platform application communication. OMG has defined the standard called the common object request broker architecture (CORBA) model. The CORBA model defines the language and a set of services that can be used to define and establish interagent communication. A manager of agents could communicate with its agents and with the user using a CORBA compliant system, but CORBA does not define any specifics that are directly related to the issue of agent management.

CUBRICON Intelligent Window Manager (Funke, Neal, \& Paul, 1993) is a prototype that defines where particular types of windows (e.g., text, graphical map, table, and form windows) should be placed on opening, or which of the open windows should be closed if there is no more screen space for another window. In designing our solution, we considered similar variables as described in Funke et al. (1993): priority of the agent; importance of the results of the agent both with keywords and with prioritized results; when the agent was initiated; given a time limit on the agent, how late is the agent; what is the current user status; and so forth.

Research presented in Zlotkin and Rosenschein (1993) addresses the issue of faimess in interaction mecharisms among agents. Without fairness, some agents can take advantage of others and the efficiency of the system will suffer. We benefitted from the research presented by Zlotkin and Rosenschein by incorporating the results into the design of the decision mechanism of our DAM.

Research reported in Maes and Kozierok (1993), Kozierok and Maes (1993), and Sheth and Maes (1993) focuses on using machine learning techniques to develop intelligent agents, rather than have the user program the agents. The authors claimed that machine learning techniques can only be used in systems where there is repetitive behavior (without it, agents will not be able to learn) and where the repetitive behavior differs depending on the user (if all users were the same, then there would only be one environment to learn). So far, we have implemented a "smart" manager in that it decides whether to report the results of various agents to the user.

\section{DECISION MECHANISM}

The principal components of the system in which the DAM would operate are the user and the multiple heterogeneous agents. The following scenario is an example of interactions of the DAM with these components. When an agent is created, the application has the ability to register the agent with the DAM. The agent reports to the DAM before notifying the user. The DAM decides whether the agent is important enough to directly report the results to the user or the agent should be filtered 
by the DAM. The DAM returns one of two results: do not filter agent (i.e., report directly to the user) or filter agent (i.e., do not report to the user). There may be a case in which the user wants to quickly see the results and then delve into them later, so the user should be able to specify that both actions take place; in other words, the user should be notified immediately and the DAM should also have a log of the situation.

In order to implement this scenario, the DAM must have the following functionality: (a) manage the different types of agents, (b) collect and manage requirements from the user, and (c) keep track of the data that the agents provide to the user and display the results of the agents in an intuitive manner. Because our primary concern is with the decision mechanism of the DAM, in the rest of this section we discuss the first of these functions. The decision mechanism of the DAM must be based on parameters representing both the user requirements and the agents that try to access the user. Because the decision mechanism is to be generic, appropriate for various agents, the parameters must be obtainable from all the agents. The following parameters have been selected based on the review of various kinds of agents (numbers in parentheses represent numerical values of the variables that are used in the computation of the importance of the agent):

- User status: bored (10), flexible (20), busy (30), do-not-disturb (50).

- Agent priority: defined by the application: Iow (1), medium (2), high (3).

- Keyword association level for a keyword found in the agent's result: general (2), associated with the agent category (3), agent specific (4).

- Keyword priority: low (1), medium (2), high (3).

- Time limit (in minutes) for processing agent's task as specified by the application (optional).

- Priority of the result (optional): low (1), medium (2), high (3).

User sfatus is a parameter that the user of the DAM can vary depending on how much responsibility the user wants to delegate to the DAM. Agent priority represents the priority of a specific application. The user will be able to specify keywords as part of the profile. The user can associate keywords at different keyword association levels. A keyword can be associated with a specific agent, to allow all instances of that agent to be associated with this keyword. Keywords can also be associated at the agent category level. This allows keywords to be associated with any agent that falls into a particular category. Finally, a keyword can be associated with all agents.

The importance level of the agent calculated by the decision mechanism will be higher for any agent reporting results that contain the defined keywords in either the message or the brief report. The weight of the keyword association will be higher for the more specific level of association. Keywords can also be assigned keyword priority levels, which are used in calculating the importance of an agent.

Agent process time limit is an optional parameter that an agent can require the user to specify. It is the time duration of processing an agent's task. The importance of the agent is increased if the agent is early and decreased if the agent is overdue with respect to this time limit. When reporting its results, the agent has the option to include a priority of the result that it gathered. This option allows agents, who can 
evaluate their own results, to pass this information to the DAM to use in its decision-making process.

Using these variables, the procedure presented in Figure 1 is used to calculate the importance value of the agent.

\section{DAM ARCHITECTURE}

To evaluate and verify the concept of the DAM, a prototype of the DAM has been developed. Because agents are required to interact with the DAM, rather than interfacing directly to the agents that are available today, we implemented an environment in which various kinds of agents can be simulated. The benefit of this

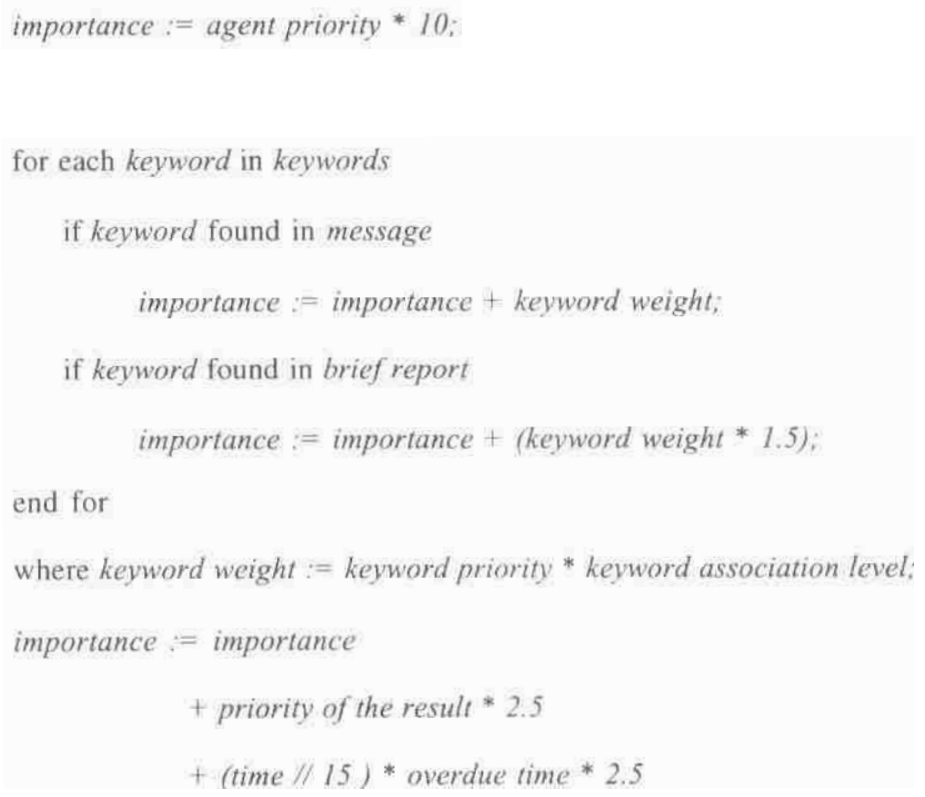

FIGURE 1 DAM decision mechanism. 


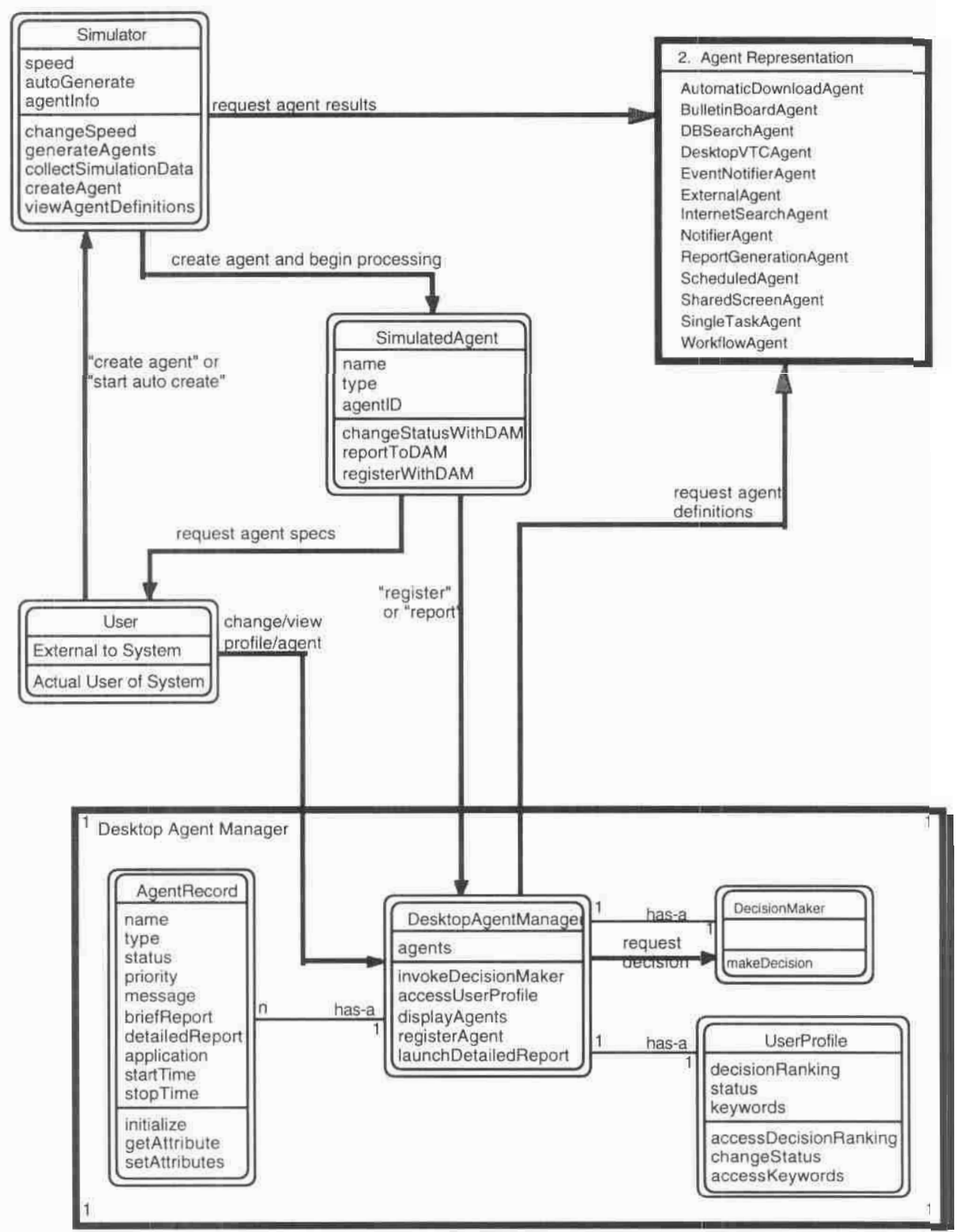

FIGURE 2 Components of the DAM architecture.

approach is one common interface for all the agents. The major components of our architecture (the object model) are shown in Figure 2. A more detailed description of the whole system can be found in Ercolini (1994). The DAM is represented in the bottom part of the figure. The upper part shows the simulator, the simulated agent, 
the repository of simulated agents, and the user. The user can interact with the simulator, the simulated agent, and the DAM.

The DAM has a User Profile, a Decision Maker, and an Agent Record as associated objects. The simulator is the object that controls the simulation environment. The simulated agent is the object that represents the external agents of the system. The Generic Agent (not shown in Figure 2) and all of its subclasses represent the different types of simulated agents and is the repository for the canned results of the simulated agents. Figure 2 is an overview of the entire object model with the Agent Representation subject collapsed into a simple list of the objects contained in the subject.

Once the agent is created, it registers with the DAM. The agent must provide the DAM with its name, its priority as assigned by the user, the agent category that it belongs to (if no category is passed, then the default value is unknown), and user-desired result time limit. The DAM retums a unique identifier to the agent that is used when reporting results or status change to the DAM. Once registered, the agent begins processing its task. When its task is completed, it sends its results to the DAM for approval.

Following the specs of the Envoy Framework (Palaniappan et al., 1992), the agent must provide a simple message to the user. The agent can also provide either a brief report to the DAM, which contains a more detailed explanation than the message, or a detailed report in the form of a file with the results of the task. The agent must also provide the application that is invoked for the user to view the results. This information can either be provided to the DAM at the time of registering or at the time of reporting results. This flexibility allows for a one-time setup if the agent always uses the same application for reporting its results as well as for an agent to be able to return results from many different applications. Another parameter the agent may report is the priority of the results of its task. Some software agents understand what they are reporting and can judge the importance of the results.

When the DAM responds, the simulated agent receives a decision. If the decision is "do not filter agent," then the simulated agent can report its results to the user. If the decision is "filter agent," the simulated agent must not report its results to the user. In either case, the simulated agent ends its task.

On request, the DAM will provide the user with the information of the currently stored agents. The DAM stores the time at which the agent was created, the name of the agent, and category of the agent. The DAM also stores the message, the brief report, and the detailed report of the agent (if applicable). The user is able to access the detailed report in the form of the native application of the data. The DAM invokes this application with the appropriate data at the request of the user. The DAM also stores what its own decision was when the agent reported (i.e., whether or not the agent was filtered). The user is able to delete agents from the stored agent information, to sort the view of the agents on several areas, like priority, category, name, time created, status, and so forth.

The simulated agent may report a change in status to the DAM. This function should not be used to change its status to completed. The agent may update its status with the following values: working, stopped, and error. The agent must also provide the unique identifier that the DAM returned when the agent registered. An optional 
parameter to this function is a simple message that may explain why the change in status occurred. Examples of the simple message for the stopped status value could contain by whom and when, and for the error status, values could contain a reason for the error. The agent should not expect a return value from the DAM in response to status change.

The agent is the initiator of communication with the DAM. If the agent does not receive a response, then the agent assumes that there is a problem and continues processing. When it finishes its task, the agent reports to the user as it normally would do. When reporting to the DAM, if the agent does not have a response from the DAM, it reports directly to the user assuming that the DAM is no longer functional.

\section{DESCRIPTION OF SIMULATION}

\subsection{Simulation Environment}

The simulation environment has been incorporated into this development as a mechanism to generate and gather data and to simulate how the DAM may act if actually put in production mode. The user of the simulation environment is the system evaluator. The simulation environment includes (ref. Figure 2) simulated agents, a simulation controller (simulator), and a simulated agent repository (agent representation). A simulated agent was described in Section 3.

The simulator allows the user to automatically generate random agents, vary the rate of simulation, and create ad hoc agents. In the automatic generation mode, agents (names and types) are selected randomly from the available agents in the simulated agent repository. Each agent is provided a random processing time and random priority. Random canned results and scenarios are also selected from a pool of agent scenarios in the repository. This information repository contains data files and associated applications so that the agent can display different types of data.

When creating ad hoc agents, the system evaluator has to specify the name of the agent. If the agent is already defined in the system, the simulation environment provides the type of agent. If the agent created is not in the predefined set, then the type of agent is automatically unknown. The user needs to provide the priority and, if desired or applicable, the time limit desired on the return of the results. Like with the automatic generation of agents, the simulator provides the canned results, including the simple message and the brief or detailed reports, and the approximate length of time that the agent takes to complete its task.

\subsection{Simulated Agents}

There are many different types of agents and many different ways in which to categorize these agents. One categorization of agents is by how or where they are invoked. Externally invoked (external) agents are generated when another user requests them. This is seen in applications today like Timbuktu, E-mail, and Desktop VTC. In these cases, some extemal entity is invoking an interruption. The DAM must be able to intercept these types of agents. Internally invoked agents can be further classified into two categories. A single task agent is created once to do a 
specific task and once it is done, it is destroyed. The other type is a kind of agent that does its task over and over, referred to as a reoccurring agent. This can be a scheduled query agent or an agent that is monitoring for a specific event and when the event occurs, the agent is activated. In our system, the software agents defined in Table 1 have been simulated. They are briefly described using the types of categories described previously.

\section{TESTING RESULTS}

For testing purposes, three sets of simulated agents were generated randomly using the simulator's Control Panel, described in detail in Ercolini (1994). The reason for generating three scenarios instead of one was to show that the performance of the DAM does not significantly depend on the selection of the variables defining scenarios. These scenarios were used at each rate of the simulation with and without the DAM activated. There was also a predefined keyword set used during the testing of the system. All other decision mechanism variables were varied randomly on all levels.

In the following figures, we present the results of our simulations. Figures 3 and 4 present the raw data collected when running the three test scenarios. These figures

\section{Table 1: Simulated Agents}

\begin{tabular}{|c|c|c|c|}
\hline Name & Today's Application & Category & Description \\
\hline Desktop VTC & ShareVision & Extemal & Provides live video teleconferencing \\
\hline agent & & & $\begin{array}{l}\text { on the desktop. Randomly } \\
\text { invoked by external user. }\end{array}$ \\
\hline $\begin{array}{l}\text { Shared screen } \\
\text { agent }\end{array}$ & $\begin{array}{l}\text { Farallon } \\
\text { Timbuktu }\end{array}$ & External & $\begin{array}{l}\text { Allows the sharing of windows on } \\
\text { the desktop. }\end{array}$ \\
\hline $\begin{array}{l}\text { Database search } \\
\text { agent }\end{array}$ & SQL Sever, Oracle & Single task & $\begin{array}{l}\text { Query for information, ad hoc query, } \\
\text { searching on specific keywords. }\end{array}$ \\
\hline $\begin{array}{l}\text { Intemet search } \\
\text { agent }\end{array}$ & $\begin{array}{l}\text { Gopher, WWW, } \\
\text { Mosaic }\end{array}$ & Single task & $\begin{array}{l}\text { Search for information on the } \\
\text { Internet. }\end{array}$ \\
\hline $\begin{array}{l}\text { Bulletin board } \\
\text { agent }\end{array}$ & $\begin{array}{l}\text { Patty Maes's } \\
\text { agents }\end{array}$ & Monitoring & $\begin{array}{l}\text { Monitor for articles to be published } \\
\text { on a bulletin board on the intemet. }\end{array}$ \\
\hline $\begin{array}{l}\text { Automatic } \\
\text { download agent }\end{array}$ & Lotus Notes & Scheduled & $\begin{array}{l}\text { Scheduled query for information. } \\
\text { Notes automatically calls another } \\
\text { Notes server and downloads any } \\
\text { additions, deletions, or changes to } \\
\text { the replicated database. }\end{array}$ \\
\hline $\begin{array}{l}\text { Report generation } \\
\text { agent }\end{array}$ & $\begin{array}{l}\text { Macros within } \\
\text { Microsoft Word } \\
\text { and Excel }\end{array}$ & Scheduled & $\begin{array}{l}\text { Automatic report generator. Word } \\
\text { and Excel can have macros that will } \\
\text { generate a periodic report given a } \\
\text { certain set of information exists. }\end{array}$ \\
\hline $\begin{array}{l}\text { Workflow notifier } \\
\text { agent }\end{array}$ & Reach Workman & Monitoring & $\begin{array}{l}\text { Sign this PR with } 24 \text { hr, review this } \\
\text { document by next Tuesday. }\end{array}$ \\
\hline $\begin{array}{l}\text { Event notifier } \\
\text { agent }\end{array}$ & IBM's IntelliAgent & Monitoring & $\begin{array}{l}\text { Budget is overrunning, schedule is } \\
\text { slipping, someone changed a file. }\end{array}$ \\
\hline
\end{tabular}




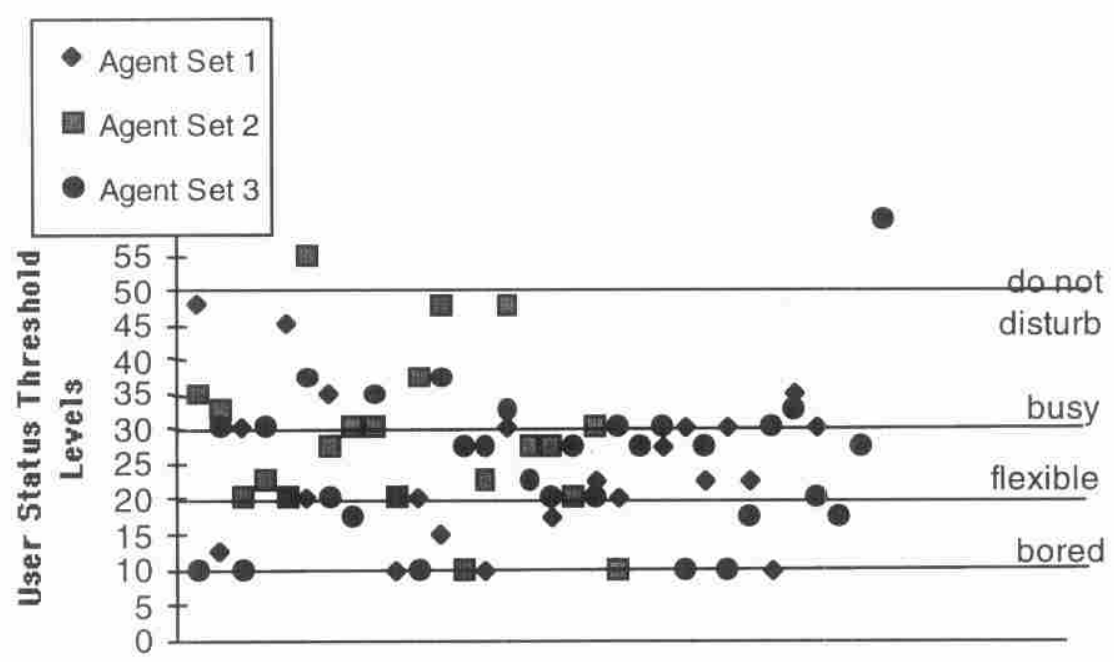

Time

FIGURE 3 User status threshold values for all agent sets.

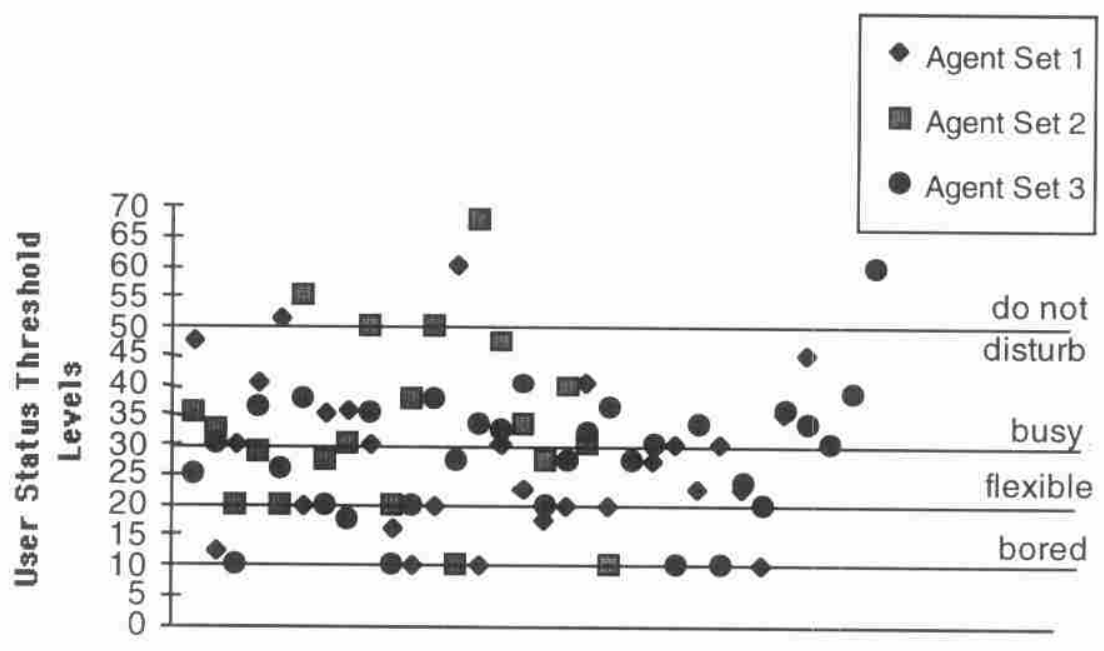

Time

FIGURE 4 User status threshold values for all agent sets using keywords.

show the default threshold values of the user status as compared to the importance values calculated for the agents. Any agent with a value that falls on or above the threshold value will not be filtered by the DAM (i.e., the agent can report its results directly to the user). The first figure is the importance values calculated without using the predefined keywords, and the second figure is the importance values calculated when using the predefined set of keywords. 


\section{ANALYSIS OF RESULTS}

Although our overall goal was to investigate the whole concept of a DAM, including the specifications, the design and the user interface, in this article we focus only on the decision mechanism of the DAM. Our primary interest was to investigate the effectiveness of the DAM with respect to its decisions on which agents and under what conditions should be filtered out and not allowed to access the user, and when they should be allowed to present their results to the user directly. Although the effectiveness of the decision mechanism can be defined as the number of agents not filtered by the decision mechanism, the real questions are (a) whether the user agrees with the DAM's decisions on which of the agents should be filtered out and which should not, and (b) whether the DAM's global policy on the number of agents that are filtered out is well synchronized with the user as represented by the user status variable (i.e., whether the user will be able to control the strictness of the filtering mechanism through this variable). To answer the first question, a human factors study is needed, including real users in real scenarios. This kind of study is beyond the scope of this article. We concentrated on the second question (i.e, on the relation between the user status and the number of filtered agents).

To assess the validity of the agent's global policy we need to understand what kind of relation should we expect, what kind of relation is "good" or "correct?" In our investigations, we assumed that the characteristic of the DAM in terms of the number of agents that are filtered should be "linear" with respect to the user status value. Linear is in quotes because user status is not a quantitative variable. Nevertheless, it is an ordinal-scale variable. Intuitively, the linearity means that if user status changes by one unit (e.g., from bored to flexible) the increase in the number of filtered agents should be the same as when user status changes from busy to do not dist urb. It also means that the number of agent interruptions should decrease as the user status level increases.

Figures 5 and 6 show the percentage of agents that were not filtered by the DAM versus the user status level. Figure 5 is for the agents running without using a keyword set and Figure 6 is for the agents using the keyword set. As expected, the

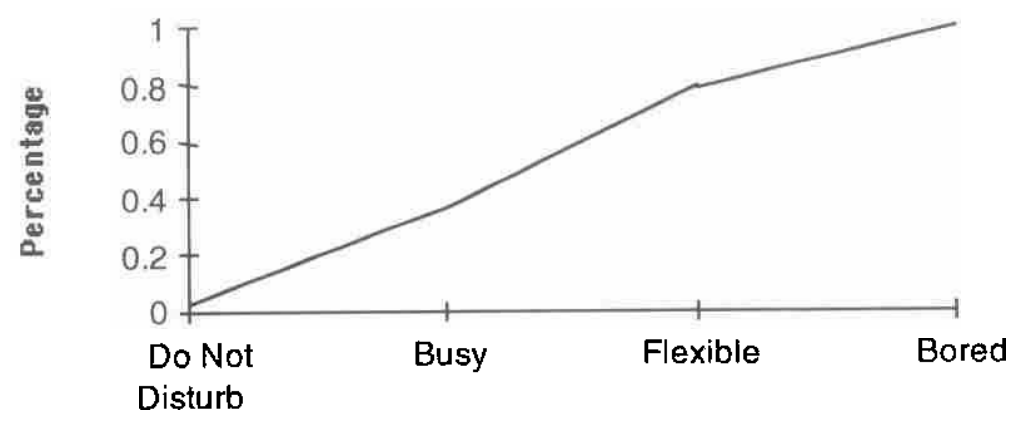

User Status Level

FIGURE 5 Average percentage of agents not filtered. 


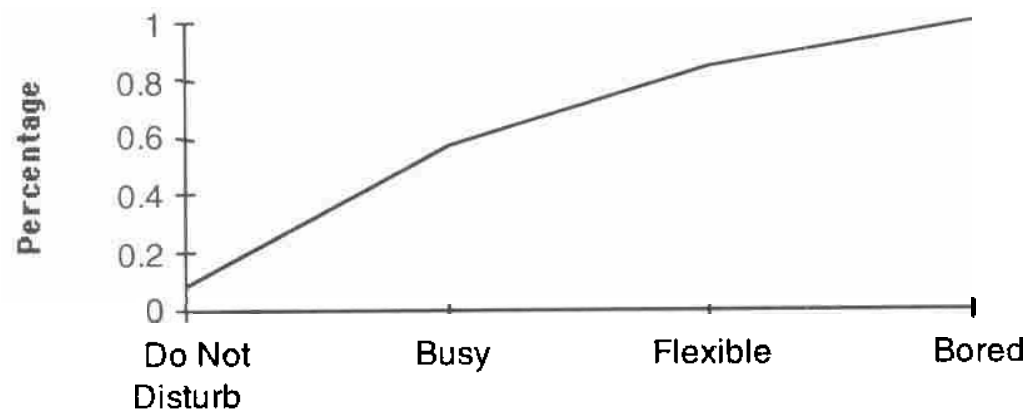

User S tatus Level

FIGURE 6 Average percentage of agents not filtered using keywords.

percentage of agents not filtered increases monotonically as the user status level moves from do not disturb to bored, approaching the number of agents generated. At the do not disturb level, a small percentage of agents are not filtered and at the bored level, all agents are not filtered. User status levels busy and bored fall in between these extremes roughly linearly. It can be concluded that the decision mechanism filters agents correctly with respect to the user status level.

In analyzing how the keywords affect the decision mechanism, using Figures 5 and 6, we can see that although the keyword capability of the decision mechanism increases the importance of agents, the increase in importance values is not constant across all agents and that keywords do not make a predictable impact. The keyword importance values increased sensitivity slightly, but not enough to conclude that they have a significant weighting in the decision mechanism.

In order to better understand the effects of the decision mechanism we analyzed the distribution of the importance values. Figures 7 and 8 show the distribution of the importance values calculated for all the agents for the test scenarios being run without and with using keywords, respectively. The distribution charts indicate that the importance values calculated by the decision mechanism are mostly in the range of 15 to 40 . This could result in uneven sensitivity of the decision mechanism with respect to user status. In our definition of the user status variable, we partially addressed this issue by scaling this variable in such a way that its value increases faster for the upper end than for the middle. This scaling can be optimized further toward the linearity of the decision mechanism characteristic.

In the next step, we investigated which parameters used for calculating the importance value of the agent are affecting the importance values. Figure 9 shows which combinations of parameters were present in the simulated data and contributed in calculating the importance value of the agents with the test case scenarios. The following legend was used in the figure for particular parameters: $P$ denotes agent priority, $R$ denotes priority of the results, $T$ denotes time limit, and $K$ denotes keywords found in the results. Only 6 of the possible 16 combinations of parameters were used for two reasons. First, the priority criteria factor $(P)$ is always present, eliminating the eight combinations that do not include P. Second, all simulated 


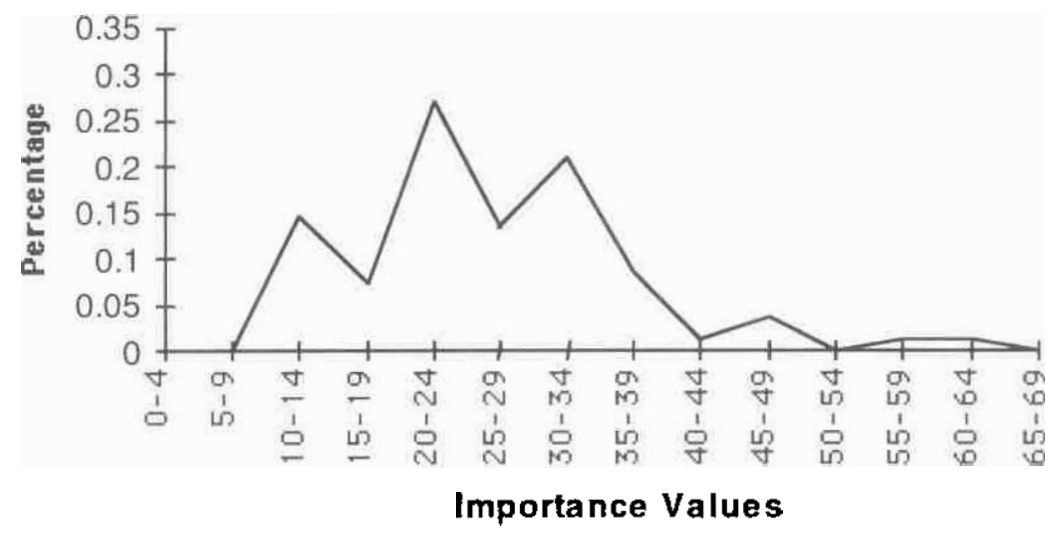

FIGURE 7 Average distribution of agent importance values

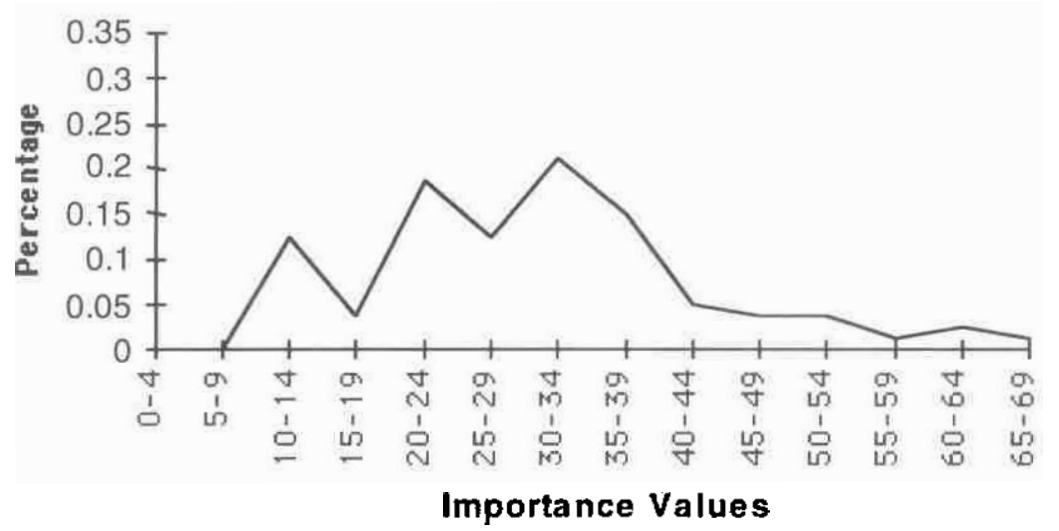

FIGURE 8 Average distribution of agent importance values using keywords.

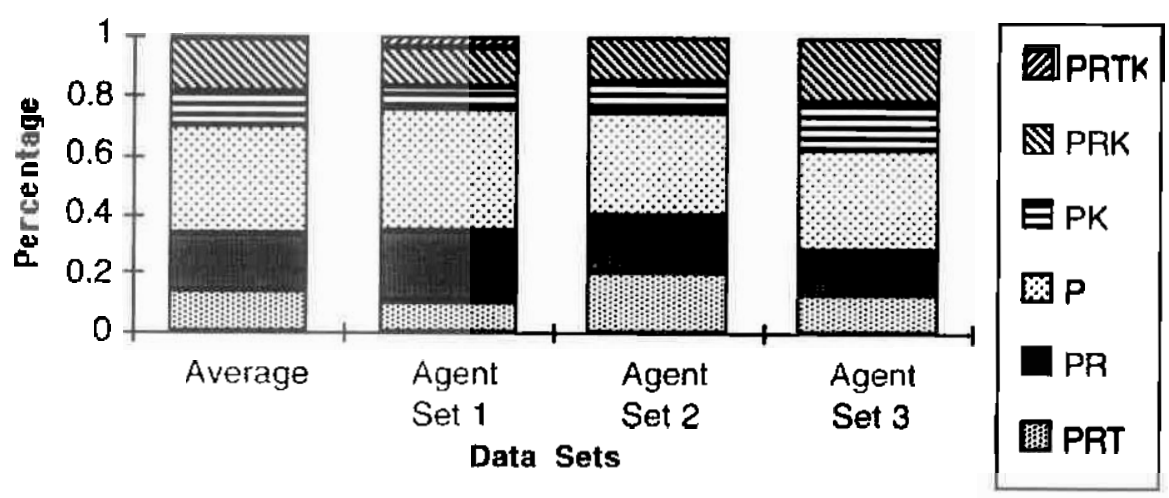

FIGURE 9 Percentage of parameters used by the decision mechanism 


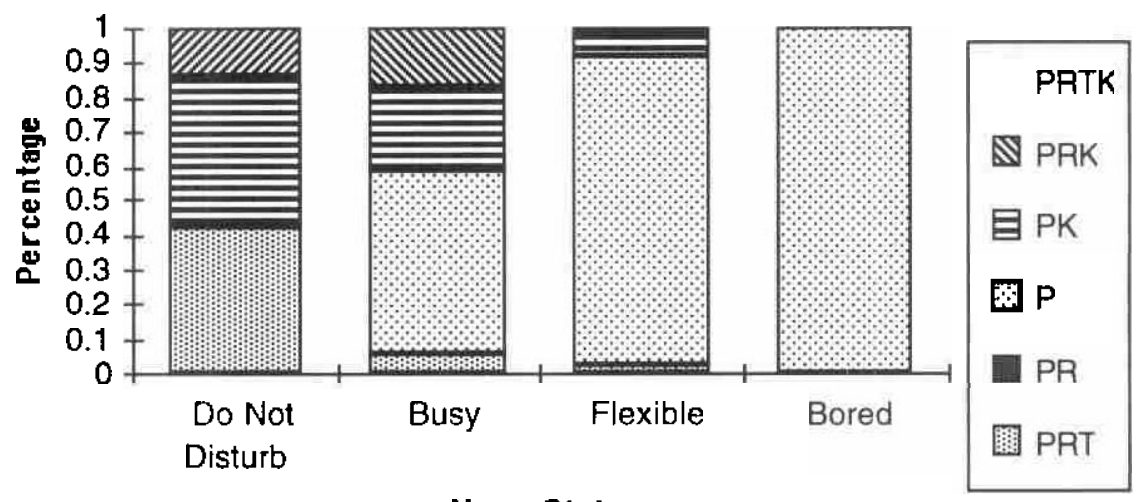

User Status

FIGURE 10 Percentage of parameters found to be critical

agents that allow the user to specify a time limit also allow the agent to prioritize its results. This eliminates the combinations that involve $T$ without $R$ (i.e., PT and PTK). This chart indicates that the combinations of parameters were essentially the same throughout all test scenarios. The leftmost bar is the average of the combinations contributing to the importance values in all three test scenarios.

Figure 10 shows the percentage of the parameters that were critical to the decision-making process for different user status levels. Critical parameters were defined as the parameters that were necessary for the calculated importance value of the agent to be equal to or higher than the user status threshold. From Figure 10, it can be seen that as the user status threshold value decreases, the priority of the agent becomes the predominant critical criteria parameter. The other parameters have less significance in determining whether the agent should be filtered or not. At the bored user status level, the only critical parameter is the priority of the agent. Even at the flexible level, priority is the predominant parameter. This concludes that the decision mechanism relies heavily on the priority of the agent.

\section{CONCLUSIONS AND FUTURE DIRECTIONS}

As more software agents are being developed in the research community as well as for commercial products, the issue of agents competing for the user's attention and agent management should be recognized. What needs to be addressed is the issue of the user being overwhelmed by the number of agents processing tasks on a single desktop computer. This article presents a possible solution to managing these agents for the user.

The goal of this research was to investigate the concept of a DAM. Toward this goal, we implemented a prototype of a DAM and a simulation environment to generate various agents and to evaluate the performance of the DAM. Overall, the DAM development achieved many of its goals. The DAM (a) captured, filtered, and managed the different types of agents; (b) collected and managed requirements from the user; and (c) displayed the results of the agents in a concise manner. 
Our primary focus was on the decision mechanism of the DAM, which makes the decision to either allow the agent to access the user or to filter the agent. Our goal was to develop a decision mechanism that can be understandable to the user and through which the user can easily control the number of agents not filtered by varying the user status variable and by using keywords. As shown in the previous analysis, the DAM performed as expected. As the user status level decreased, the number of agents not filtered increased proportionally.

All testing was performed both without using keywords and using a certain keyword set. We found that although keywords increased the importance values, the increase was not constant across all agents, because it depends on the keyword set defined by the user and the number of matches to the agent results. Because no one keyword set can be considered the "typical" keyword set chosen by any user, the only way to truly judge whether keywords have a major impact is to perform a user evaluation of the system with many users and keyword sets.

The analysis of the decision mechanism that we performed resulted in several suggestions for the design of this mechanism. We concluded that, depending on the values of user status, the sensitivity of the decision mechanism can become biased for or against some of the parameters. For instance, when the threshold value is low (i.e, bored or flexible), the priority of the agent is the only parameter that is critical. When setting user status to bored, the user should understand that all agents will not be filtered because the priority of the agent is the only parameter taken into account. Unless we provide the user with the feedback showing them why the decisions were made, the user may never understand the reasoning behind the decision mechanism.

We showed that the characteristic of the agent can be optimized by the user toward a linear characteristic through adjusting the scaling of the user status parameter. Another possibility is to allow the user to change the weights for particular parameters. The next level of flexibility would be to allow the user to change the decision mechanism algorithm. This is a much more involved solution and it would require providing the user with a programming language in which to set the decision mechanism rules. The next step would be to incorporate machine learning into the DAM. The DAM would learn by watching how the user interacts with the agents that exist and generate its own rules.

Although all of these solutions are feasible from the technical point of view, the final recommendation requires more research involving users. Any change in the decision mechanism leads to a learning curve for the user. For instance, in both the rule-based and learning approaches, the DAM would have to have a language for defining new rules. The user would have to have a good understanding of this language in order to be able to either change or learn the new rules. Also, although a DAM with learning capabilities is desirable, the user of such an agent must be provided with a mechanism to control such an agent. The main decision now would be when to allow the agent to make independent decisions. To further analyze the effectiveness of the DAM, a user study should be performed in which all of these possibilities are evaluated. 


\section{REFERENCES}

Adler, M., Durfee, E., Huhns, M., Punch, W., \& Simoudis, E. (1992, Summer). AAAI Workshop on Cooperation Among Heterngeneous Intelligent Agents. AI Magazine, pp. 39-42.

Avouris, N., Van Liedekerke, M.r Lekkas, G., \& Hall, L. (1993). User interface design for cooperating agents in industrial process supervision and control applications. Inlermational Jotrmal of Man-Machine Studies, 38, 873-890.

Bird, S. (1993). Toward a taxonomy of multi-agent systems. International foumal of Man-Machine Studies, 39, 689-704.

Ercolini, D. A. G. (1994). An architecture for a desktop agent manager. Unpublished master's thesis, Northeastern University, Boston.

Funke, D., Neal, J., \& Paul, R. (1993). An approach to intelligent automated window management. International Journal of Math-Machine Stutits, 38, 949-983.

Kozierok, R., \& Maes, P. (1993). A learning interface agent for scheduling meetings. In International workshop for infelligent user interfaces (pp. 81-88). Orlando, FL: ACM Press.

Maes, P., \& Kozierok, R. (1993). Learning interface agents. Proceedings of AAAI '93, 459-465.

Miley, M. (1993). Agent technology: The fine line between smart design and intelligent software. MacWEEK, 7, 41-45.

Palaniappan, M., Yankelovich, N., Fitzmaurice, G., Loomis, A., Haan, B., Coombs, J., \& Meyrowitz, N. (1992). The envoy framework: An open architecture for agents. ACM Trmusactions on Information Systems, 10, 233-264.

Sheth, B., \& Maes, P. (1993, March). Evolving agents for personalized information filtering. Paper. presented at the 9th IEEE Conference on Artificial Intelligence for Applications, Orlando, FL.

Streeter, A. (1993). Open Sesamel summons agents to automate tasks. MacWEEK, 7, 1-2.

Tessler, F. (1993). Magnet 1.0 intelligent agent-based utility. Macworld, 10, 220.

Zlotkin, G., \& Rosenschein, J. (1993). A domain theory for task oriented negotiation. Proceedings of IICAI'93, 416-422. 